\title{
Heterostructures Produced from Nanosheet-Based Inks
}

\author{
F. Withers, ${ }^{\dagger}$ H. Yang, ${ }^{\ddagger}$ L. Britnell, ${ }^{\dagger}$ A. P. Rooney, ${ }^{\S}$ E. Lewis, ${ }^{\S}$ A. Felten,, C. R. Woods, ${ }^{\dagger}$ \\ V. Sanchez Romaguera, ${ }^{\ddagger}$ T. Georgiou, ${ }^{\dagger}$ A. Eckmann, ${ }^{\dagger}$ Y. J. Kim, ${ }^{\dagger}, \perp$ S. G. Yeates, ${ }^{\ddagger}$ S. J. Haigh, ${ }^{\S}$ A. K. Geim, \\ K. S. Novoselov, ${ }^{\dagger}$ and C. Casiraghi* ${ }^{*}+$ \\ ${ }^{\dagger}$ School of Physics and Astronomy, ${ }^{\ddagger}$ School of Chemistry, ${ }^{\S}$ School of Materials, and ${ }^{\#}$ Manchester Centre for Mesoscience and \\ Nanotechnology, University of Manchester, Oxford Road, Manchester, M13 9PL, United Kingdom \\ "Research Centre in Physics of Matter and Radiation (PMR), Université de Namur, B-5000 Namur, Belgique \\ ${ }^{\perp}$ Department of Chemistry, College of Natural Sciences, Seoul National University, Seoul, 151-747, Korea
}

\section{Supporting Information}

\begin{abstract}
The new paradigm of heterostructures based on two-dimensional (2D) atomic crystals has already led to the observation of exciting physical phenomena and creation of novel devices. The possibility of combining layers of different $2 \mathrm{D}$ materials in one stack allows unprecedented control over the electronic and optical properties of the resulting material. Still, the current method of mechanical transfer of individual

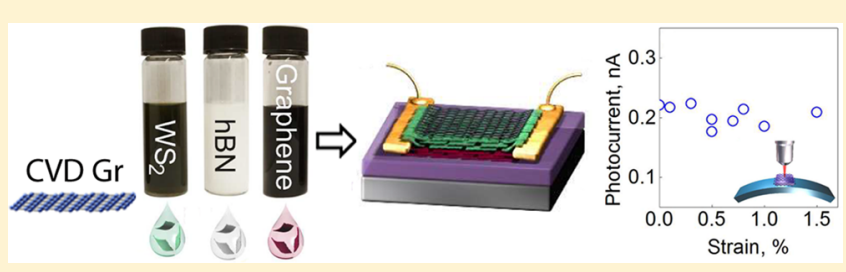
$2 \mathrm{D}$ crystals, though allowing exceptional control over the quality of such structures and interfaces, is not scalable. Here we show that such heterostructures can be assembled from chemically exfoliated $2 \mathrm{D}$ crystals, allowing for low-cost and scalable methods to be used in device fabrication.
\end{abstract}

KEYWORDS: Graphene, 2D crystals, inks, devices, heterostructures, flexible electronics

\begin{abstract}
A large variety of $2 \mathrm{D}$ atomic crystals isolated in the recent years offer a rich platform for the creation of heterostructures ${ }^{1-3}$ which combine several of these materials in one stack. Since, collectively, this class of materials covers a very broad range of properties, the obtained heterostructures can be tuned to focus on particular phenomena or be used for specific applications ${ }^{4-15}$ (or even to perform multiple functions). Still, up to now all vertical heterostructures have been produced by micromechanical cleavage ${ }^{16}$ of threedimensional layered crystals with subsequent dry transfer ${ }^{4,6}$ of each crystal layer. While this technique allows one to achieve extremely high quality stacks, ${ }^{17}$ it certainly cannot be applied to the production of such heterostructures on a large scale. So, alternative methods, compatible with mass production and that do not require the use of clean rooms and expensive techniques such as lithography, should be utilized to bring the attractive qualities of such systems to real-life applications.

One approach is based on the use of liquid-phase exfoliation (LPE) to produce dispersions of various $2 \mathrm{D}$ crystals. ${ }^{18-20}$ One can then use such inks to deposit platelet layers of different materials sequentially by standard low-cost fabrication techniques (drop- and spray-coatings, roll-to-roll transfer, inkjet printing, ${ }^{21}$ etc.). LPE has already been used as a mass-scale approach for production of 2D crystals and offers several advantages for cost reduction and scalability. One of the most important advantages of LPE is that the same method can be used to create inks made of nanosheets of different 2D crystals, covering a large variety of properties. Furthermore, this technique is compatible with low-cost and flexible substrates, so it is expected to have a big impact on the new generation of
\end{abstract}

flexible electronics and photovoltaics. ${ }^{11}$ Also, despite the small size of the flakes and the use of surfactants, the $2 \mathrm{D}$ material flakes still demonstrate similar properties to those of the largescale $2 \mathrm{D}$ crystals obtained by micromechanical cleavage, ${ }^{19,20}$ thus creating stable, long-lasting inks with well-defined properties. Such LPE-produced inks and heterostructures have already been used to make simple devices such as sensors for hydrogen evolution reaction ${ }^{22}$ and planar photovoltaic devices. ${ }^{23}$ However, here we will show that more complex, multifunctional, and flexible devices, based on completely different physical principles, can be created.

The purpose of this work is to show the proof of concept of a promising low-cost technology, ${ }^{24}$ suitable for mass-production of devices based on heterostructures, which can be applied to make devices of arbitrary complexity. Here we take this concept to the ultimate level, combining a number of different materials in a controlled vertical stack. We present several examples of such heterostructures created by depositing LPE 2D crystals via drop-casting, inkjet printing, and vacuum filtration. In particular, we make use of graphene $(\mathrm{Gr})$, transition metal dichalcogenides (TMDC, such as $\mathrm{WS}_{2}$ and $\mathrm{MoS}_{2}$ ), and hexagonal-boron nitride (h-BN) inks (details on the production and characterization of the dispersions can be found in the Supporting Information). These crystals have been selected because of their complementary electronic and dielectric

Received: April 11, 2014

Revised: May 23, 2014

Published: May 28, 2014 
properties, ranging from the high transparency and conductivity of graphene, ${ }^{25}$ to the high optical absorption of TMDC ${ }^{26}$ and the high transparency and resistivity of h-BN. ${ }^{14,27,28}$ We fabricated and tested different types of devices with the following general structure: BGr/barrier/TGr, where TGr and $\mathrm{BGr}$ refer to top and bottom graphene electrodes, respectively (Figure 1). Here we demonstrate that such devices can act as

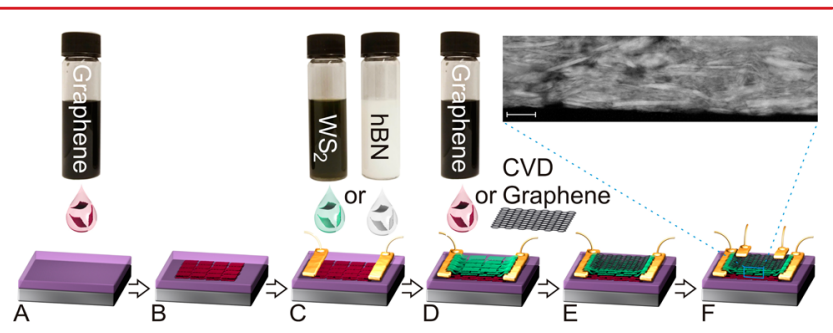

Figure 1. Schematic of a general heterostructure device fabrication process made by using $2 \mathrm{D}$-crystal inks. Last panel shows the final device and the cross-sectional STEM HAADF image of the $\mathrm{WS}_{2}$ thin film. The scale bar is $35 \mathrm{~nm}$.

(i) tunnelling transistors, ${ }^{11,12,29}$ where tunnelling between $\mathrm{TGr}$ and BGr through a barrier (typically made of TMDC) is controlled by a back gate; (ii) photovoltaic devices, where light absorbed in the barrier (TMDC) is converted into photocurrent through TGr and BGr; (iii) in-plane transistors, where TGr is used as a gate and the barrier as a gate dielectric to control the in-plane current in BGr.

Results and Discussion. Figure 1 shows a schematic of a general process used to fabricate such devices: graphene ink is deposited on a $\mathrm{Si} / \mathrm{SiO}_{2}$ substrate (Figure $1 \mathrm{~A}$ ) to fabricate the bottom electrode (Figure 1B). Then TMDC or h-BN inks are used to fabricate a thin film on top of the bottom electrode (Figure 1C). Cross-sectional scanning transmission electron microscopy (STEM) high-angle annular dark field (HAADF) imaging has been used to access the quality and the stacking of the laminates in our inks (Figure 1F). The TGr is usually composed of CVD (chemical vapor deposition), LPE, or mechanically exfoliated graphene (Figure 1D,E) to ensure sufficient optical transparency. In the case of mechanically exfoliated graphene, the deposition was done by using a dry transfer method. ${ }^{4,6}$

Three low-cost and scalable methods have been used for the deposition of LPE 2D crystals: drop-casting, inkjet printing, and vacuum filtration (Figure 2). We utilized different types of dispersions (depending of the deposition method used): from $N$-methylpyrrolidone-based (NMP) dispersions ${ }^{19,20}$ to aqueous dispersions obtained by mixed solvents ${ }^{30}$ or by using pyrene derivatives. $^{31}$ Compared to generally used NMP and DMF

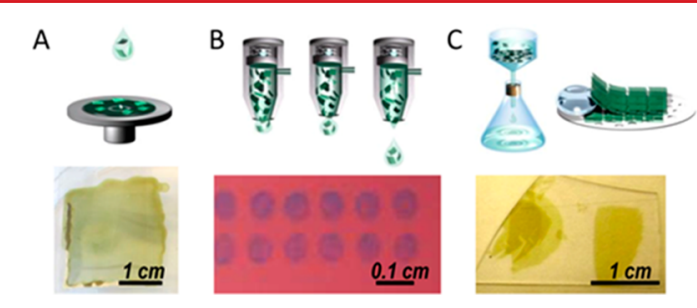

Figure 2. Schematic representations of deposition methods for LPE of 2D atomic crystals and optical micrographs of deposited films: (A) drop-casting on glass; (B) inkjet printing on $\mathrm{Si} / \mathrm{SiO}_{2}(300 \mathrm{~nm})$; (C) vacuum filtration and fishing on glass. dispersions of graphene and other 2D crystals, such aqueous dispersions offer a much faster drying rate and contains a lower amount of stabilizer, which is essential when using our deposition methods (for instance in postprinting processing). Aqueous dispersions are also much more environmentally friendly.

In the specific case of vertical heterostructure the quality of the film is determined by the amount of defects and pin-holes. Therefore, it is essential to characterize and compare the coatings produced with different methods (Supporting Information). For the purposes of our relatively small (typically submillimeter) device, we observed that the three methods give very similar results. Raman spectroscopy does not show any strong changes between TMDC films obtained with the three methods. Furthermore, all of the methods allow one to produce pinhole-free films: (i) inkjet printing allows controlling the shape of the films (stripes, dots, etc.) and to remove pinholes by printing several times over the same feature; ${ }^{21}$ (ii) drop casting and spray coating allow covering large area and pinhole free films can be obtained by using highly concentrated dispersions; (iii) filtering and fishing allow coverage of a smaller area, compared to drop casting, but the pinhole density can be controlled by repeating the transfer on the same area several times (typically, the density of the pinholes is strongly reduced after two transfers; see the Supporting Information). The presence of pinholes can immediately be detected by the very low tunnelling resistance of our tunnel junctions. At the same time, the finite size and stiffness of TMDC and graphene flakes makes the device insensitive to small pin-holes due to the "bridging" effect. Further details of the methods can be found in the Supporting Information.

We start with tunnelling junctions and tunnelling transistors which have a structure of $\mathrm{Si} / \mathrm{SiO}_{2} / \mathrm{BGr} / \mathrm{WS}_{2} / \mathrm{TGr}\left(\mathrm{MoS}_{2}\right.$-based devices demonstrate similar characteristics; see the Supporting Information). Tunnelling junctions may have both BGr and TGr produced by either of the methods mentioned above, whereas tunnelling transistors require exactly monolayer graphene to be used as $\mathrm{BGr}$ in order not to screen the gate voltage (and as such are prepared from CVD or mechanically exfoliated graphene). The current-bias voltage $\left(I-V_{\mathrm{b}}\right)$ characteristics for our devices are strongly nonlinear (Figure 3). As expected, the zero-bias conductivity goes down as the thickness of $\mathrm{WS}_{2}$ layer increases (Figure 3A). The uncertainty in the thickness of the layer (RMS roughness $\sim 3 \mathrm{~nm}$ ) prevents us from any quantitative analysis of the scaling behavior. Zero-bias conductivity also decreases dramatically with decreasing temperature (Figure 3B). Such a strong temperature dependence suggests an excitation mechanism for charge carrier generation, either from the graphene electrodes (in this case the tunnelling barrier is the Schottky barrier between graphene and $\mathrm{WS}_{2}$ ) or from the impurity band in $\mathrm{WS}_{2}$ (a strong impurity band is expected due to the large fraction of edges in our nanocrystals of $\mathrm{WS}_{2}$ ). We would like to note that a variation in the tunnelling barrier thickness leads to effectively a range of tunnelling barriers connected in parallel and can contribute to the strong temperature dependence. The strong increase in the current for $V_{\mathrm{b}}>1 \mathrm{~V}$ even at low temperatures (Figure 3B inset) suggests overbarrier transport between graphene and $\mathrm{WS}_{2}$.

For devices where $\mathrm{BGr}$ was made of monolayer graphene, gating with the Si back gate (through $300 \mathrm{~nm} \mathrm{SiO}_{2}$ ) is possible (Figure $3 \mathrm{C}$ ). The density of states in monolayer graphene around the Dirac point is very low, which allows manipulation of the work function of graphene and the electric field 

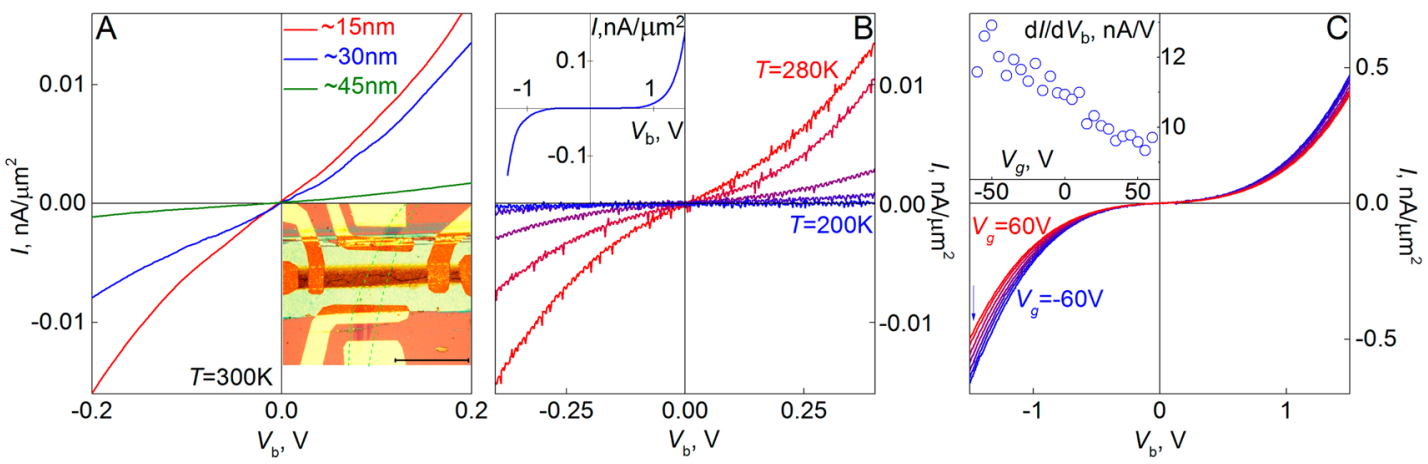

Figure 3. (A) $I-V_{\mathrm{b}}$ curves for $\mathrm{Si} / \mathrm{SiO}_{2} / \mathrm{BGr} / \mathrm{WS}_{2} / \mathrm{TGr}$ heterostructures with different thickness of $\mathrm{WS}_{2} \cdot V_{\mathrm{g}}=0 \mathrm{~V}$. Inset: optical micrograph of one of our devices. Boundaries of $\mathrm{BGr}$ (yellow, produced by drop coating) and $\mathrm{TGr}$ (green, mechanically exfoliated few layer graphene) are marked by dashed lines. The whitish (when on $\mathrm{Si} / \mathrm{SiO}_{2}$ ) or reddish (when on gold contacts) area is LPE $\mathrm{WS}_{2}$. Scale bar $=100 \mu \mathrm{m}$. (B) Temperature dependence (from $T=200 \mathrm{~K}$ (blue) to $T=280 \mathrm{~K}$ (red) in $20 \mathrm{~K}$ steps) of the $I-V_{\mathrm{b}}$ characteristics of a BGr $/ \mathrm{WS}_{2} / \mathrm{TGr}$ device $\left(\mathrm{WS}_{2}\right.$ thickness $\sim 30$ $\mathrm{nm}$, here we used mechanically exfoliated graphene as BGr and TGr). Inset: $I-V_{\mathrm{b}}$ for the same device at $T=4.2 \mathrm{~K} . V_{\mathrm{g}}=0 \mathrm{~V}$. (C) $I-V_{\mathrm{b}}$ characteristics for the same device at different $V_{\mathrm{g}}$ (from $V_{\mathrm{g}}=-60 \mathrm{~V}$ (blue) to $V_{\mathrm{g}}=60 \mathrm{~V}$ (red) in $20 \mathrm{~V}$ steps). $T=300 \mathrm{~K}$. Inset: Differential conductivity of the same device at $V_{\mathrm{b}}=-1.5 \mathrm{~V}$ (marked by the blue arrow in the main panel) as a function of $V_{\mathrm{g}}$.

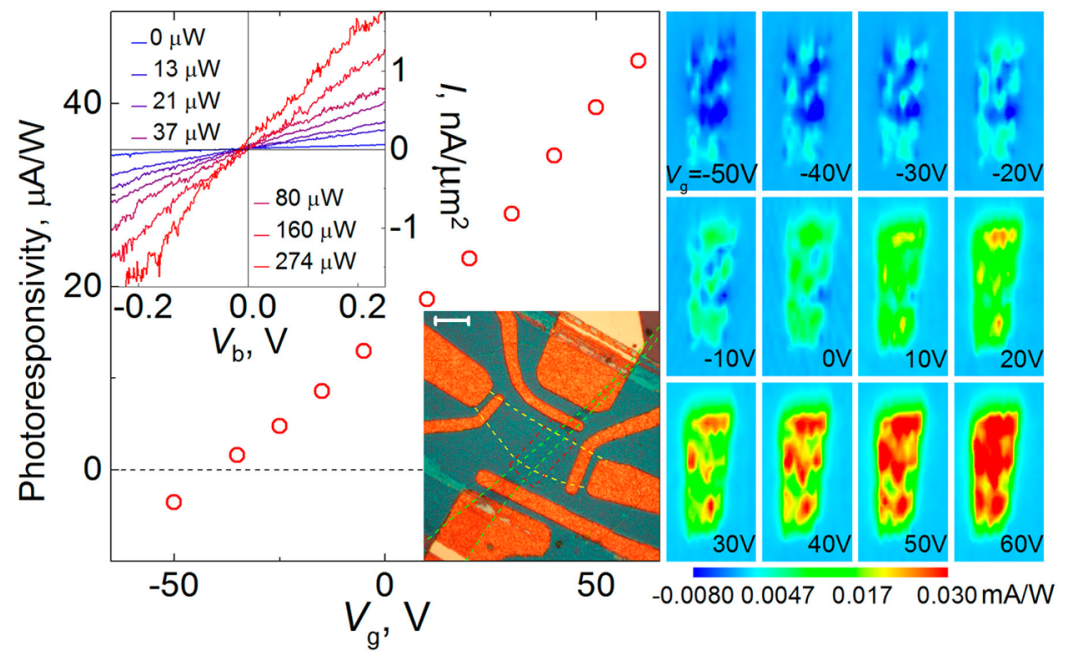

Figure 4. Left panel: Photoresponsivity (zero-bias photocurrent) of a $\mathrm{Si} / \mathrm{SiO}_{2} / \mathrm{BGr} / \mathrm{WS}_{2} / \mathrm{TGr}$ device as a function of $V_{\mathrm{g}}$. Each point is obtained by averaging the zero bias photocurrent maps (right panel). The photocurrent changes sign at $V_{\mathrm{g}} \approx-40 \mathrm{~V}$, indicating reversing in the direction of the built-in electric field. Top inset: $I-V_{\mathrm{b}}$ characteristics of the device at different laser powers. Bottom inset: optical micrograph of one of our devices. Boundaries of $\mathrm{BGr}$ (yellow) and $\mathrm{TGr}$ (green) are marked by dashed lines. Both BGr and TGr are produced by transferring mechanically exfoliated graphene. The greenish (when on $\mathrm{Si} / \mathrm{SiO}_{2}$ ) or reddish (when on gold contacts) area is LPE $\mathrm{WS}_{2}$. Scale bar $=10 \mu$ m. Right panel: spatial maps of the zero bias photocurrent for the same device, taken from the area marked by red rectangular in the bottom insert to the left panel at different values of $V_{\mathrm{g}}$. The width of each map is $10 \mu \mathrm{m}$. Incident power of $56 \mu \mathrm{W}$ and laser energy is $1.96 \mathrm{eV}$ (see Supporting Information for different excitation energies).

penetrating to the $\mathrm{WS}_{2}$ barrier with the gate voltage. The zerobias resistance is not sensitive to the back gate voltage $V_{\mathrm{g}}$ applied, whereas the current in the nonlinear region demonstrates a $30 \%$ modulation when $V_{\mathrm{g}}$ is swept between -60 and $60 \mathrm{~V}$. The fact that the gate voltage mostly affects the nonlinear part of the $I-V_{\mathrm{b}}$ dependence indicates that the changes in the current are mostly due to the changes in the relative position of the Fermi energy with respect to the top of the valence band in $\mathrm{WS}_{2}$ (as it has been previously suggested for tunnelling transistors produced from monocrystalline $\mathrm{WS}_{2}{ }^{12}$ ) and not due to the gating of $\mathrm{WS}_{2}$. The gating of $\mathrm{WS}_{2}$, which would result in modification of the shape of the tunnelling barrier (making it triangular), is not very efficient in LPE samples because of the large impurity band (due to edges), which screens the electric field. Note that from the slope of the conductivity versus $V_{\mathrm{g}}$ one can conclude that hole transport through the valence band of $\mathrm{WS}_{2}$ dominates the current, contrary to the conclusion of ref 12 where monocrystalline $\mathrm{WS}_{2}$ has been used. This might be due to the fact that the Fermi level in small flakes of $\mathrm{WS}_{2}$ is pinned close to the valence band by the edge states.

Similar structures have been used for photovoltaic applications. Again, in the main text we limit ourselves to $\mathrm{Si} /$ $\mathrm{SiO}_{2} / \mathrm{BGr} / \mathrm{WS}_{2} / \mathrm{TGr}$ type heterostructures (see results for $\mathrm{MoS}_{2}$ in the Supporting Information). Upon illumination, electron-hole pairs generated mostly in the TMDC layer (due to its high optical absorption ${ }^{13}$ ) can decay into separate electrodes (provided there is an electric field to separate the charges), producing a photovoltage. ${ }^{13}$ Under illumination the $I-V_{\mathrm{b}}$ characteristics become increasingly linear (Figure 4, inset), demonstrating that in this regime the current is dominated by the photoexcited carriers. Also, finite photocurrent has been observed even at zero bias voltage (Figure 4 
inset to left panel), demonstrating that such structures can be indeed used as photovoltaic devices.

We recorded zero-bias photocurrent as a function of the position of the laser spot (less than $1 \mu \mathrm{m}$ in diameter) on the device by using a $100 \times$ microscope objective. Zero-bias photocurrent maps, taken at different back gate voltages, demonstrate that the photocurrent is produced only in the regions where all three layers (BGr, TMDC, and TGr) overlap. We notice that the edges of the sample provide a slightly offset gate dependence of the photocurrent, which we attribute to the effect of environmental doping of the TMDC not covered by TGr. Similar to the case of the transistor, the back gate voltage controls the value and the direction of the electric field across $\mathrm{WS}_{2}$ and thus the magnitude and the polarity of the photocurrent (Figure 4). For the largest electric field across $\mathrm{WS}_{2}\left(\right.$ at $\left.V_{\mathrm{g}}=60 \mathrm{~V}\right)$ used in these measurements, we achieved photoresponsivity values of $\sim 0.1 \mathrm{~mA} / \mathrm{W}$.

The efficiency could still be increased by using larger flakes; electron and hole scattering and localization on the impurities and edges are reduced, which, in turn, would reduce the contribution of the recombination mechanisms. ${ }^{32} \mathrm{We}$ would like to stress that our devices do not require exactly monolayer TMDC to be used, which simplifies the procedure even further. Using even thicker flakes (by reducing the sonication time) means that those would also be larger laterally and allow more efficient charge transfer between the layers, thus allowing for more efficient $\mathrm{e}-\mathrm{h}$ separation. Also, the use of thick TMDC flakes ensures that the band structure of TMDC used has a indirect band gap, ${ }^{33,34}$ thus reducing the probability of recombination. Note that the photoabsorption for TMDC (per layer) practically does not change with the number of layers. $^{13}$

Although the photoresponsivity of our devices is significantly smaller than that obtained in current state of the art photovoltaic devices ${ }^{35}$ or in similar heterostructures based on monocrystalline $\mathrm{WS}_{2}{ }^{13}$ the advantage of our structures is that they can be produced by a variety of low-cost and scalable methods and are compatible with flexible substrates (note that the use of CVD graphene as a back electrode for solar cell application is compatible both with the flexible substrates and with this low cost method). To this end we fabricated PET/ $\mathrm{BGr} / \mathrm{WS}_{2} / \mathrm{TGr}$ heterostructures on a flexible PET substrate (thickness $0.2 \mathrm{~mm}$ ) (Figure $5 \mathrm{~A}$ ). We tested two different methods for sample fabrication: $\mathrm{BGr}$ and $\mathrm{WS}_{2}$ layers were produced by either drop-casting or vacuum filtering (with subsequent wet transferring) of the respective LPE dispersion. Both layers were shaped into strips by mechanical removal of the unnecessary material (Figure 5A). We used CVD graphene as TGr to achieve maximum optical transparency. A four-point bending rig was utilized to apply uniaxial strain to the heterostructure (Figure 5D).

As in the previous experiment we scanned a laser across the sample while simultaneously recording the photocurrent (Figure 5B,C). The photocurrent is only observed when illuminating the area where all three layers $\left(\mathrm{BGr}, \mathrm{WS}_{2}\right.$, and TGr) overlap. After bending, some local variation in the photocurrent was detected. However, the overall pattern (Figure 5B, C), the integral value of the photocurrent (Figure $5 \mathrm{E}$ ), and the overall resistance of the device (Figure $5 \mathrm{~F}$ ) remain practically independent of the strain, demonstrating the possibility to use such heterostructures for flexible electronics.

Finally, we demonstrate a different type of heterostructure where LPE h-BN is used as a gate dielectric. The dielectric

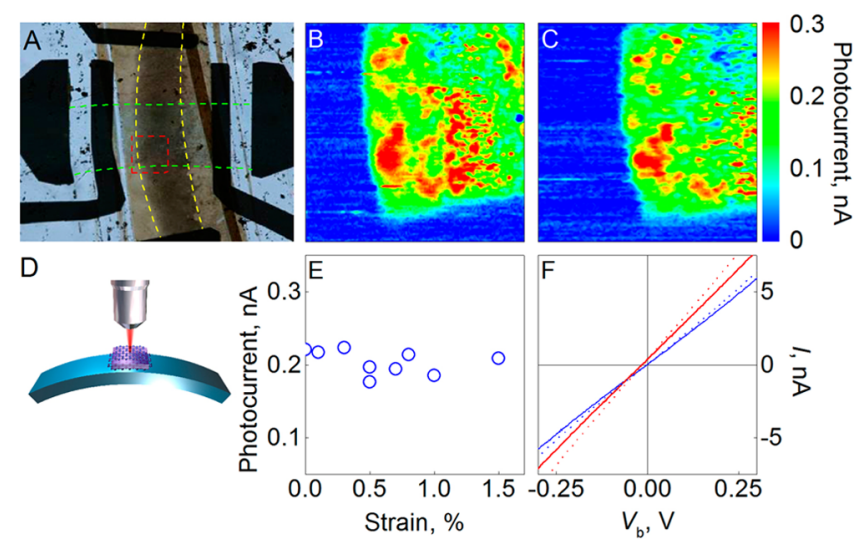

Figure 5. (A) Optical micrograph of a LPE PET/BGr/ $\mathrm{WS}_{2} / \mathrm{TGr}$ heterostructure. The yellow dotted lines indicate the boundaries of LPE BGr; the green dotted lines CVD TGr; the red square shows the area investigated by photocurrent mapping (size $70 \mu \mathrm{m} \times 70 \mu \mathrm{m}$ ). The brownish stripe which covers the $\mathrm{BGr}$ is $60 \mathrm{~nm} \mathrm{LPE} \mathrm{WS}$. Photocurrent maps $(70 \mu \mathrm{m} \times 70 \mu \mathrm{m})$ taken at an incident power of $190 \mu \mathrm{W}$ and energy of $1.96 \mathrm{eV}$ at two different curvatures: $0 \mathrm{~mm}^{-1}$ (B, corresponds to zero strain) and $0.15 \mathrm{~mm}^{-1}$ (C, corresponds to $1.5 \%$ strain). (D) Schematic representation of our bending setup. (E) Average photocurrent obtained from the photocurrent maps as a function of the applied strain. (F) $I-V_{\mathrm{b}}$ characteristics with (red) and without (blue) illumination for the strained (solid curves) and unstrained (dashed curves) cases. The illumination (power $190 \mu \mathrm{W}$ ) was focused into a $\sim 1 \mu \mathrm{m}^{2}$ spot.

properties of h-BN, ${ }^{19,28}$ added to its excellent chemical and thermal stability, mechanical and thermal properties, ${ }^{1}$ make $h-$ $\mathrm{BN}$ thin films a promising dielectric alternative in the next generation of nanodevices. ${ }^{36}$ Here we tested $\mathrm{Si} / \mathrm{SiO}_{2} / \mathrm{BGr} / \mathrm{h}$ $\mathrm{BN} / \mathrm{Au}$ devices, where LPE h-BN (prepared through filtering of a h-BN suspension, with subsequent transfer of the h-BN paper from the filter to the device) served as transparent dielectric between the channel (BGr, CVD graphene) and the gate $(\mathrm{Au})$ (Figure 6A, inset). We also used mechanically exfoliated singleand few-layer CVD and LPE graphene as a top electrode.

The resistivity of the BGr channel as a function of top gate voltage $V_{\mathrm{gt}}$ is presented in Figure 6A. The contour plot of the resistivity as a function of $V_{\mathrm{g}}$ and $V_{\mathrm{gt}}$ demonstrates the usual resistivity maximum shifting across a diagonal of the plot (Figure 6B, the dashed line). The slope of the line allows us to establish the ratio of the capacitances to $\mathrm{Si}$ and top gate (here we ignore the finite compressibility of $2 \mathrm{D}$ electron gas in graphene). Knowing the thickness of h-BN from the AFM study (600 nm for this particular sample) allows us to estimate the effective dielectric constant of LPE h-BN to be $\sim 1.5$. The significant deviation from the bulk value $(\sim 4$, as established in recent tunnelling experiments ${ }^{37}$ ) is due to lose packing of h-BN laminates. This low value of the dielectric constant of the LPE $\mathrm{h}-\mathrm{BN}$ could be an advantageous property when considering its incorporation in densely packed electronic elements, where loss needs to be kept to a minimum. Indeed air gaps in conventional insulators are deliberately induced to reduce the overall effective dielectric constant. ${ }^{38,39}$ Knowing the capacitance to the top gate allows us to estimate the mobility of the BGr to be of the order of $3 \times 10^{3} \mathrm{~cm}^{2} / \mathrm{V} \cdot \mathrm{s}$, which is typical of CVD graphene. ${ }^{4}$ This clearly indicates that deposition of LPE h-BN does not deteriorate the properties of graphene. We have also tested the breakdown voltage for our LPE h-BN (Supporting Information), which turned out to be $0.25 \mathrm{~V} / \mathrm{nm}$. This is 

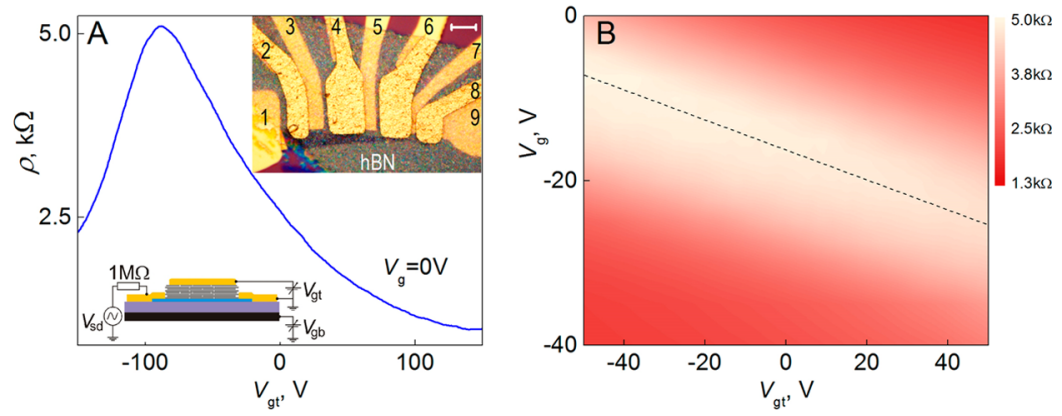

Figure 6. (A) Resistivity as a function of $V_{\mathrm{g}}$ for one of our $\mathrm{Si} / \mathrm{SiO}_{2} / \mathrm{BGr} / \mathrm{h}-\mathrm{BN} / \mathrm{Au}$ device (schematic in the bottom inset). T= $300 \mathrm{~K}$. Top inset: optical micrograph of one of our device. BGr is contacted by odd-numerated contacts and gated though LPE h-BN (seen as colorful, partly transparent areas) by even-numerated contacts. Scale bar $=10 \mu \mathrm{m}$. (B) Resistivity as a function of $V_{\mathrm{g}}$ and $V_{\mathrm{gt}}$. The dashed line indicates the position of the resistivity maximum. $T=300 \mathrm{~K}$.

comparable (or better) than traditionally used inkjet printed dielectric ${ }^{40}$ or the dielectric strength of spattered films. ${ }^{41}$ This demonstrates that LPE h-BN can be used as a dielectric for transparent, flexible transistor applications.

Conclusions. Demonstrated examples show that inks based on $2 \mathrm{D}$ atomic crystals are suitable for printable, flexible, and transparent electronics. Moreover, the combinations of different inks allow for the creation of complex heterostructures, which might be suitable for multifunctional applications. Although many of the heterostructures created still underperform in comparison with the benchmark structures, their versatility, low cost, the simplicity of the technology, and unique properties (e.g., flexibility and transparency) might prove beneficial for some types of devices. We foresee that multifunctional applications might gain the most, as a large number of very different $2 \mathrm{D}$ crystals could be combined in one stack. At the same time, some applications, such as the use of h$\mathrm{BN}$ as a high-performance dielectric material, are already in the mature state. Furthermore, the possibility of fine-tuning the properties of the inks, by varying the size and thickness of the flakes as well as the type of solvent, will increase the range of functionalities of the resulting heterostructures and devices even further.

Materials and Methods. The dispersions were produced via sonication of the original crystals in different solvents (NMP, water, and water/ethanol mixture). The films were produced by drop-casting, inkjet printing, and filtering and were characterized by Raman spectroscopy, AFM, TEM, and XPS. Photocurrent measurements were performed by irradiating the active area of the devices with a laser of energy $1.96 \mathrm{eV}$. A $100 \times$ objective with numerical aperture 0.60 was used to focus the spot to less than $1 \mu \mathrm{m}$ diameter. The power was accurately measured using a Thorlabs PM100 power meter with sensitivity of $10 \mathrm{nW}$. A voltage drop over a known resistor placed in series with the photodiode was used to calculate the photocurrent. We then utilized a piezo stage with a step of $100 \mathrm{~nm}$ to move the sample under the laser beam. The stage position was linked to the photocurrent measurements in order to obtain spatial information on the photocurrent. The external quantum efficiency (EQE) is calculated as the number of charge carriers collected by the contacts to the number of incident photons, $\mathrm{EQE}=(h f / e)\left(i_{\mathrm{pc}} / P\right)$, where $h$ is Planck's constant, $f$ is the frequency of the incident photon, $e$ is the electron charge, $i_{\mathrm{pc}}$ is the photocurrent at zero bias, and $P$ is the incident laser power (see Supporting Information for detailed analysis of the photovoltaic performance).

\section{ASSOCIATED CONTENT}

\section{Supporting Information}

Detailed information on material preparation, characterization, and fabrication of the devices, including additional devices. This material is available free of charge via the Internet at http:// pubs.acs.org.

\section{AUTHOR INFORMATION}

\section{Corresponding Author}

*E-mail: cinzia.casiraghi@manchester.ac.uk.

\section{Notes}

The authors declare no competing financial interest.

\section{ACKNOWLEDGMENTS}

This work was supported by The Royal Society, U.S. Army, European Science Foundation (ESF) under the EUROCORES Programme EuroGRAPHENE (GOSPEL), European Research Council, and EC under the Graphene Flagship (contract no. CNECT-ICT-604391). Y.-J.K.'s work was supported by the Global Research Laboratory (GRL) Program (2011-0021972) of the Ministry of Education, Science and Technology, Korea. F.W. acknowledges support from the Royal Academy of Engineering; A.F. is a FRS-FNRS Research Fellow.

\section{REFERENCES}

(1) Geim, A. K.; Grigorieva, I. V. Nature 2013, 499, 419.

(2) Novoselov, K. S.; et al. Rev. Mod. Phys. 2011, 83, 837.

(3) Novoselov, K. S.; et al. Proc. Natl. Acad. Sci. U.S.A. 2005, 102, 10451

(4) Dean, C. R.; et al. Nat. Nanotechnol. 2010, 5, 722.

(5) Dean, C. R.; et al. Nat. Phys. 2011, 7, 693.

(6) Ponomarenko, L. A.; et al. Nat. Phys. 2011, 7, 958.

(7) Gorbachev, R. V.; et al. Nat. Phys. 2012, 8, 896.

(8) Ponomarenko, L. A.; et al. Nature 2013, 497, 594.

(9) Dean, C. R.; et al. Nature 2013, 497, 598.

(10) Hunt, B.; et al. Science 2013, 340, 1427.

(11) Britnell, L.; et al. Science 2012, 335, 947.

(12) Georgiou, T.; et al. Nat. Nanotechnol. 2013, 8, 100.

(13) Britnell, L.; et al. Science 2013, 340, 1311.

(14) Britnell, L.; et al. Nano Lett. 2012, 12, 1707.

(15) Britnell, L.; et al. Nat. Commun. 2013, 4, 1794.

(16) Novoselov, K. S.; et al. Science 2004, 306, 666-17.

(17) Haigh, S. J.; et al. Nat. Mater. 2012, 11, 764.

(18) Blake, P.; et al. Nano Lett. 2008, 8, 1704.

(19) Hernandez, Y.; et al. Nat. Nanotechnol. 2008, 3, 563.

(20) Coleman, J. N.; et al. Science 2011, 331, 568.

(21) Torrisi, F.; et al. ACS Nano 2012, 6, 2992.

(22) Yang, J.; et al. Angew. Chem., Int. Ed. 2013, 52, 13751. 
(23) Finn, D. J.; et al. J. Mater. Chem. C 2014, 2, 925.

(24) Krebs, F. C. Sol. Energy Mater. Sol. Cells 2009, 93, 394.

(25) Nair, R. R.; et al. Science 2008, 320, 1308.

(26) Wang, Q. H.; Kalantar-Zadeh, K.; Kis, A.; Coleman, J. N.; Strano, M. S. Nat. Nanotechnol. 2012, 7, 699.

(27) Gorbachev, R. V.; et al. Small 2011, 7, 465.

(28) Lee, G. H.; et al. Appl. Phys. Lett. 2011, 99, 243114.

(29) Meric, I.; et al. Proc. IEEE 2013, 101, 1609.

(30) Zhou, K. G.; Mao, N. N.; Wang, H. X.; Peng, Y.; Zhang, H. L. Angew. Chem., Int. Ed. 2011, 50, 10839.

(31) Yang, H.; et al. Carbon 2013, 53, 357.

(32) Kulyuk, L.; et al. Phys. Rev. B 2005, 72, 075336.

(33) Mak, K. F.; Lee, C.; Hone, J.; Shan, J.; Heinz, T. F. Phys. Rev. Lett. 2010, 105, 136805.

(34) Kumara, A.; Ahluwalia, P. K. Eur. Phys. J. B 2012, 85, 186.

(35) Polman, A.; Atwater, H. A. Nat. Mater. 2012, 11, 174.

(36) Osada, M.; Sasaki, T. Adv. Mater. 2012, 24, 210.

(37) Ponomarenko, L. A.; et al. J. Appl. Phys. 2013, 113, 136502.

(38) Volksen, W.; Miller, R. D.; Dubois, G. Chem. Rev. 2010, 110, 56.

(39) Maex, K.; et al. J. Appl. Phys. 2003, 93, 8793.

(40) Mengel, M.; Nikitin, I. Microelectron. Eng. 2010, 87, 593.

(41) Bartzsch, H.; et al. Phys. Status Solidi A 2009, 206, 514. 